\title{
Effective description of dark matter as a viscous fluid
}

\author{
Stefan Floerchinger ${ }^{1}$, Mathias Garny ${ }^{2}$, Nikolaos Tetradis ${ }^{2,3, \star}$, and Urs Achim Wiedemann ${ }^{2}$ \\ ${ }^{1}$ Institut für Theoretische Physik, Universität Heidelberg, 69120 Heidelberg, Germany \\ ${ }^{2}$ Theoretical Physics Department, CERN, CH-1211 Genève 23, Switzerland \\ ${ }^{3}$ Department of Physics, University of Athens, Zographou 157 84, Greece
}

\begin{abstract}
Treating dark matter at large scales as an effectively viscous fluid provides an improved framework for the calculation of the density and velocity power spectra compared to the standard assumption of an ideal pressureless fluid. We discuss how this framework can be made concrete through an appropriate coarse-graining procedure. We also review results that demonstrate that it improves the convergence of cosmological perturbation theory.
\end{abstract}

\section{Introduction}

The calculation of the power spectrum of cosmological fluctuations for stochastic initial conditions is an important and challenging task. It provides the basis for analyzing data on the large-scale structure of the universe and can lead to constraints on the parameters of the cosmological model. The spectrum can be computed through $N$-body simulations. However, these are CPU-intensive, while they do not provide an intuitive understanding of the underlying physical processes. The alternative option is an analytical treatment, which amounts to solving the collisionless Vlasov-Poisson equation perturbatively in the density contrast for appropriate classes of initial conditions [1]. The use of perturbative techniques in this context is complicated by the growth of non-linearities at short length scales. Within the strongly non-linear regime, velocity dispersion and virialization becomes important.

The inability of analytical methods to describe the short scales reliably suggests to reformulate cosmological perturbation theory based on an effective description, applicable only above some length scale. The description should contain effective parameters which absorb the effect of short-scale perturbations that are 'integrated out' [2-4]. In the conventional description of dark matter as a pressureless ideal fluid, only the lowest moments of the phase-space distribution are taken into account. Enlarging this framework by including higher moments results in viscous transport coefficients that parametrize a non-ideal stress tensor for the dark matter. In the following we present heuristic arguments that support the suggestion that an efficient fluid description of dark matter must allow for such non-ideal terms. The role of the effective viscosity and sound velocity is to account for the interaction of large-scale fluctuations (with wavenumbers $\left.k<k_{m}\right)$ with the short-scale ones $\left(k>k_{m}\right)$ that are not followed explicitly in a fluid description limited to $k<k_{m}$. In our analysis the UV contributions are incorporated in the large-scale theory at the level of a one-loop approximation. Going beyond one-loop can be achieved through renormalization-group techniques [7,8]. The approximation that

^e-mail: ntetrad@phys.uoa.gr 
we review here provides an intuitive and rather simple framework, while it still resolves the main deficiency of standard perturbation theory (SPT), namely the strong dependence on the short-scale dynamics that are out of the reach of analytical techniques. We review the calculation of the density power spectrum presented in ref. [6] in the context of the coarse-grained theory. We also present results for the velocity and cross spectra within the same framework.

\section{The relevant scales}

Our approach to the problem of cosmological matter perturbations assumes the presence of two scales: the momentum scale $k_{\Lambda}$ at which a fluid description becomes feasible, and the scale $k_{m}<k_{\Lambda}$ at which the description can be based on a small number of effective parameters. In practice we can take $k_{\Lambda} \sim 1-3 \mathrm{~h} / \mathrm{Mpc}$, corresponding to length scales $\sim 3-10 \mathrm{Mpc}$, and $k_{m} \sim 0.5-1 \mathrm{~h} / \mathrm{Mpc}$, corresponding to length scales $\sim 10-20 \mathrm{Mpc}$. At the scale $k_{m}$ the density contrast is of order 1 , while above the scale $k_{\Lambda}$ it is much larger than 1 and the dynamics involves shell crossing, making a fluid description unfeasible. The description at $k_{m}$ must include viscosity terms arising either through the coarse-graining of the scales $k>k_{m}$ (effective viscosity), or fundamental dark matter interactions (fundamental viscosity). The scales $k>k_{\Lambda}$ correspond to virialized structures, which are expected to have a negligible effect on long-distance dynamics [5]. It seems reasonable then to expect that the effective viscosity results mainly from the integration of the modes with $k_{m}<k<k_{\Lambda}$. The form of the linear power spectrum (which falls off roughly as $k^{-3}$ ) is consistent with this expectation: the effective viscosity at $k_{m}$ is dominated by the modes with $k$ slightly above this value, while the scales $k>k_{\Lambda}$ give a negligible contribution. This efficient decoupling of deep UV modes is also supported by numerical evidence from $N$-body simulations as well as analytical arguments based on non-perturbative relations of response functions $[9,10]$.

There are several reasons for introducing the scale $k_{m}$ and distinguishing it from $k_{\Lambda}$ :

- In the absence of dark-matter interactions, the form of the effective terms beyond the perfect-fluid description (such as effective viscosity) at the scale $k_{m}$ is in a certain sense universal. They do not depend strongly on the initial conditions at $k_{\Lambda}$, but only on $k_{m}$. If fundamental interactions exist, the fundamental viscosity should vary only little between $k_{\Lambda}$ and $k_{m}$, so that it can be estimated at $k_{m}$ without loss of accuracy.

- The main role of the scale $k_{m}$ is to act as an UV cutoff for perturbative corrections in the largescale theory, thus eliminating contributions of dubious validity, arising from the modes near and above $k_{\Lambda}$. This effect is seen clearly in the good convergence of our results, in contrast to standard perturbation theory [6].

- The scale $k_{m}$ can be lowered even further than the range that we are considering, if one would like to focus on the effective theory for length scales much larger than the range of baryon acoustic oscillations (BAO). In such a case, the renormalization-group improvement of the effective description is crucial [8].

\section{Estimate of the effective viscosity}

The validity of the fluid description for cold dark matter cannot be established similarly to systems close to thermal equilibrium. The crucial elements supporting this description are the small darkmatter velocity and the finite age of the universe, during which dark matter particles can drift over a finite distance, much smaller than the Hubble radius. A more quantitative discussion of this point 
is given in ref. [2]. In the absence of dark-matter interactions, one starts from the Vlasov-Poisson equations and writes the particle phase-space density as

$$
f(\mathbf{x}, \mathbf{p}, \tau)=f_{0}(p)\left[1+\delta_{f}(\mathbf{x}, p, \hat{\mathbf{p}}, \tau)\right],
$$

with $\tau$ the conformal time. The Fourier modes of the perturbation are then expanded in terms of Legendre polynomials $P_{n}$ :

$$
\delta_{f}(\mathbf{k}, p, \hat{\mathbf{p}}, \tau)=\sum_{n=0}^{\infty}(-i)^{n}(2 n+1) \delta_{f}^{[n]}(\mathbf{k}, p, \tau) P_{n}(\hat{\mathbf{k}} \cdot \hat{\mathbf{p}}),
$$

with unit vectors $\hat{\mathbf{k}}, \hat{\mathbf{p}}$. The Vlasov-Boltzmann equation implies a hierarchy of evolution equations for the higher moments:

$$
\frac{d \delta_{f}^{[n]}}{d \tau}=k v_{p}\left[\frac{n+1}{2 n+1} \delta_{f}^{[n+1]}-\frac{n}{2 n+1} \delta_{f}^{[n-1]}\right], \quad n \geq 2,
$$

with $v_{p}=p /$ am the particle velocity. If the typical value of $v_{p}$ is sufficiently small, the higher moments can be neglected.

A quantitative constraint can be obtained if one assumes that the particle velocity is of the order of the fluid velocity $v$ at small length scales. We emphasize that this assumption is valid only for cold dark matter, for which the particle velocity is induced by the gravitational fields generated by the growth of large-scale structure. The assumption is supported by the estimated typical velocities of dark matter particles in galaxy haloes. ${ }^{1}$ At the comoving momentum scale $k$ we expect $[\theta / \mathcal{H}]^{2} \sim k^{3} P^{L}(k)$, with $P^{L}(k)$ the linear power spectrum evaluated on the growing mode, $\theta=\overrightarrow{\mathbf{k}} \cdot \overrightarrow{\mathbf{v}}$ the velocity divergence, and $\mathcal{H}=(1 / a)(d a / d \tau)$. The linear spectrum scales roughly as $k^{-3}$ above a scale $\sim k_{m}$, so that $k^{3} P^{L}(k)$ is roughly constant, with a value of order 1 today. Its time dependence is given by $D_{L}^{2}$, with $D_{L}$ the linear growth factor. We expect then that the maximal particle velocity can be identified with the fluid velocity at the scale $k_{m}$, and is roughly

$$
v_{p} \sim \frac{\mathcal{H}}{k_{m}} D_{L}
$$

The time $\tau$ available for the higher moments to grow is $\sim 1 / \mathcal{H}$. Therefore, the dimensionless factor characterizing the growth of higher moments is $k v_{p} / \mathcal{H} \sim D_{L} k / k_{m}$. Scales with $k \gg k_{m} / D_{L}$ require the use of the whole Boltzmann hierarchy and cannot be treated through a fluid description that takes into account only the lowest moments. In practice, the validity of the fluid description extends beyond $k_{m}$ at all times, as the initial values of the higher moments are much smaller than 1 , while $D_{L} \leq 1$. Therefore, on large scales, cold dark matter can be described as a cosmological fluid, not because microscopic interaction rates are sufficiently large to maintain local thermal equilibrium, but because the lifetime of the universe is too short for dark matter to deviate strongly from local equilibrium.

The above picture can lead to an estimate of the effective viscosity $\eta$ that must be attributed to the dark-matter fluid because of the particle velocities that deviate from the uniform motion. In general, we expect $\eta /(\rho+p) \sim l_{\text {free }} v_{p}$, with $l_{\text {free }}$ the mean free path. For the effective viscosity we can estimate $l_{\text {free }} \sim v_{p} / H$, with $H=\mathcal{H} / a$. In this way we obtain for the kinematic viscosity of the dark-matter fluid

$$
v_{\text {eff }} \mathcal{H}=\frac{\eta_{\text {eff }}}{\rho_{m} a} \mathcal{H} \sim l_{\text {free }} v_{p} H \sim \frac{\mathcal{H}^{2}}{k_{m}^{2}} D_{L}^{2},
$$

\footnotetext{
${ }^{1}$ Due to the cosmological expansion, the massive particles that constitute the cold dark matter have negligible velocity originating in their thermal motion before freeze-out. Warm dark-matter particles would have much larger velocities, which makes the application of the fluid description to this case problematic.
} 
where we have neglected the pressure. The numerical value is small, of the order of $10^{-6}$ today, and even smaller at higher redshifts. However, viscosity plays a significant role in the context of the coarse-grained theory.

We can also estimate the viscosity induced by possible fundamental interactions between the darkmatter particles. For interacting dark matter, with number density $n$, mass $m$ and cross section $\sigma$, the mean free path is $l_{\text {free }} \sim 1 /(n \sigma)$. We expect then fundamental viscosity

$$
v_{\text {fund }} \mathcal{H}=\frac{\eta_{\text {fund }}}{\rho_{m} a} \mathcal{H} \sim l_{\text {free }} v_{p} H \sim \frac{1}{n \sigma} \frac{H^{2}}{k_{m}} a D_{L} \sim \frac{m}{\sigma} \frac{8 \pi G_{N}}{3 k_{m}} \frac{a D_{L}}{\Omega_{m}} .
$$

The time dependence, given by the last factor, indicates that the fundamental viscosity drops very quickly at early times. We point out that the above estimate is valid only for cold dark matter, for which the typical particle velocity is induced by the late-time growth of structure. For warm dark matter, the typical velocity and the resulting viscosity would evolve differently with time.

We note that that the fundamental viscosity of cold dark matter does not grow indefinitely for decreasing $\sigma$. The mean free path of dark matter particles cannot exceed $v_{p} / H \sim a D_{L} / k_{m}$. As a result, for values of $\sigma$ such that $1 /(n \sigma)$ exceeds $a D_{L} / k_{m}$, the particle scattering has no effect. This implies that the fundamental viscosity cannot take values larger than roughly $\left(\mathcal{H}^{2} / k_{m}^{2}\right) D_{L}^{2}$, and is always subleading or comparable to the effective viscosity.

As a final remark, we would like to emphasize that the constraints derived in refs. [11-13] for the non-ideal properties of dark matter are not applicable to the case of cold dark matter that we are considering. In these works the viscosity and sound velocity are assumed to stay constant or even grow with redshift, in contrast to the redshift-dependence indicated by our eqs. (5) and (6). In our picture, the particle velocity is induced by the growth of structure and is a late-time phenomenon. The assumptions of refs. [11-13] can by justified only within a warm-dark-matter scenario, in which the particles have velocities originating in their primordial motion.

\section{One-loop determination of the effective viscosity}

For the construction of the coarse-grained theory we follow the Wilsonian approach. A detailed description, including a discussion of the renormalization-group flow of the coarse-grained theory, is given in ref. [8]. The main idea is that the effective parameters of the large-scale theory result from the integration of the UV modes. For the problem at hand, the effective viscosity and sound velocity of the dark-matter fluid are given by one-loop expressions that depend on the dimensionful scale

$$
\sigma_{d m}^{2}(\tilde{\eta})=\frac{4 \pi}{3} \int_{k_{m}}^{\infty} d q P^{L}(q, \tilde{\eta})=\frac{4 \pi}{3} D_{L}^{2}(\tilde{\eta}) \int_{k_{m}}^{\infty} d q P^{L}(q, 0)
$$

with $\tilde{\eta}=\ln a=-\ln (1+z)$ and $D_{L}(\tilde{\eta})$ the linear growth factor: $\delta(\mathbf{k}, \tilde{\eta})=D_{L}(\tilde{\eta}) \delta(\mathbf{k}, 0)$ on the growing mode. For a spectrum that scales as $1 / k^{3}$, the integral is dominated by the region near $k_{m}$.

A precise definition of the effective couplings can be given through the propagator of theory. One first defines the doublet

$$
\left(\begin{array}{c}
\varphi_{1}(\mathbf{k}, \tilde{\eta}) \\
\varphi_{2}(\mathbf{k}, \tilde{\eta})
\end{array}\right)=\left(\begin{array}{c}
\delta_{\mathbf{k}}(\tilde{\eta}) \\
-\frac{\theta_{\mathbf{k}}(\tilde{\eta})}{\mathcal{H}}
\end{array}\right),
$$

and the propagator

$$
G_{a b}\left(\mathbf{k}, \tilde{\eta}, \tilde{\eta}^{\prime}\right) \delta^{(3)}\left(\mathbf{k}-\mathbf{k}^{\prime}\right)=\left\langle\frac{\delta \varphi_{a}(\mathbf{k}, \tilde{\eta})}{\delta \varphi_{b}\left(\mathbf{k}^{\prime}, \tilde{\eta}^{\prime}\right)}\right\rangle
$$


The power spectrum is defined as

$$
\left\langle\varphi_{a}(\mathbf{k}, \tilde{\eta}) \varphi_{b}\left(\mathbf{k}^{\prime}, \tilde{\eta}\right)\right\rangle=\delta^{(3)}\left(\mathbf{k}+\mathbf{k}^{\prime}\right) P_{a b}(\mathbf{k}, \tilde{\eta}) .
$$

We concentrate on an Einstein-de Sitter (EdS) background, for which $D_{L}(\tilde{\eta})=\exp (\tilde{\eta})$ and the linear propagator for the growing mode in the perfect-fluid theory is

$$
g_{a b}\left(\tilde{\eta}-\tilde{\eta}^{\prime}\right)=\frac{e^{\tilde{\eta}-\tilde{\eta}^{\prime}}}{5}\left(\begin{array}{ll}
3 & 2 \\
3 & 2
\end{array}\right)
$$

The one-loop contribution to the low-energy propagator with wavenumber $k \rightarrow 0$, coming from modes with $q \geq k_{m}$, is given by

$$
\delta g_{a b}\left(\mathbf{k}, \tilde{\eta}, \tilde{\eta}^{\prime}\right)=-k^{2} e^{\tilde{\eta}-\tilde{\eta}^{\prime}} \sigma_{d m}^{2}(\tilde{\eta})\left(\begin{array}{cc}
\frac{61}{350} & \frac{61}{525} \\
\frac{27}{50} & \frac{9}{25}
\end{array}\right) \simeq-k^{2} e^{\eta} \sigma_{d m}^{2}(\tilde{\eta}) \frac{3}{50}\left(\begin{array}{cc}
3 \cdot 0.968 & 2 \cdot 0.968 \\
9 & 6
\end{array}\right),
$$

where we have taken the limit $\tilde{\eta} \gg \tilde{\eta}^{\prime}$ for the second equality. On the other hand, the linear propagator of a theory with small kinematic viscosity and sound velocity of the form

$$
\nu \mathcal{H}=\frac{\eta}{\rho_{m} a} \mathcal{H}=\beta_{v} e^{2 \tilde{\eta}} \frac{\mathcal{H}^{2}}{k_{m}^{2}}, \quad c_{s}^{2}=\frac{\delta p}{\delta \rho}=\frac{3}{4} \beta_{s} e^{2 \tilde{\eta}} \frac{\mathcal{H}^{2}}{k_{m}^{2}},
$$

contains a contribution

$$
\delta g_{a b}(\mathbf{k}, \tilde{\eta})=-\frac{k^{2}}{k_{m}^{2}}\left(\beta_{v}+\beta_{s}\right) \frac{e^{3 \tilde{\eta}}}{45}\left(\begin{array}{ll}
3 & 2 \\
9 & 6
\end{array}\right)
$$

in addition to the perfect-fluid term (11), where we have kept only the leading contribution for $\eta \gg \eta^{\prime}$.

We can now derive precise expressions for the effective parameters of the large-scale theory, by identifying the linear contribution to the propagator (14) for the viscous theory, with the one-loop correction (12) of the perfect-fluid propagator. This can be achieved with $1 \%$ accuracy, and we find

$$
\beta_{s}+\beta_{v}=\frac{27}{10} k_{m}^{2} \sigma_{d m}^{2}(0)
$$

It must be emphasized that there are no free parameters in our approach, as the effective couplings are uniquely determined. Moreover, the deep UV region, which is out of the reach of perturbation theory, gives a negligible contribution to the integral of eq. (7), consistently with the expectation of decoupling of virialized structures. The dominant contribution comes from the region slightly above $k_{m}$, for which perturbation theory is expected to give reliable results.

By matching heuristically viscous fluid dynamics to the one-loop contribution (14), effective couplings were explored in [6]. This procedure can be obtained as a limiting case from the renormalization-group formulation of Ref. [8]. The latter approach also allows one to break the degeneracy between $\beta_{v}$ and $\beta_{s}$ [8]. The numerical results that we present below depend only on the sum $\beta_{v}+\beta_{s}$ to a very good approximation.

\section{Density and velocity power spectra}

The procedure that we outlined in the previous section for an EdS background can be generalized to the $\Lambda \mathrm{CDM}$ case with an appropriate change of variables [6]. We present here results for the density, velocity and mixed power spectra for a large-scale viscous theory with parameters determined 

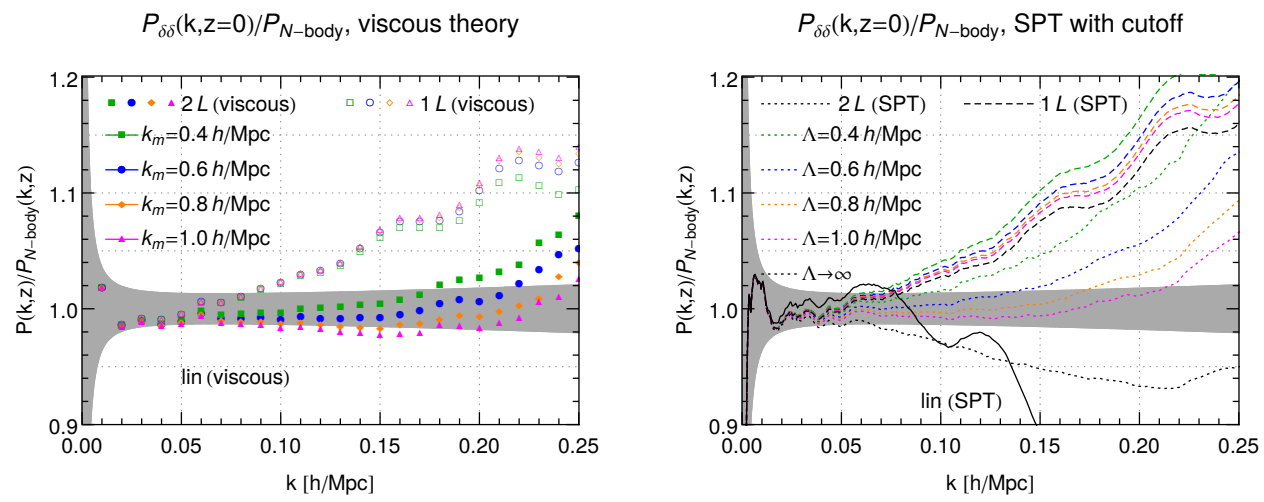

Figure 1. Density power spectrum at redshift $z=0$, normalized to the $N$-body power spectrum [14]. The left panel shows the results obtained in the viscous description, for various values of the scale $k_{m}$. The coloured lines show the linear spectra, the open and filled symbols the one- and two-loop spectra. For comparison, the right panel shows the corresponding one- and two-loop results in standard perturbation theory. Here $\Lambda$ is a sharp cutoff in $k$-space. The grey shaded region shows the estimated uncertainty of the $N$-body simulation. Figure taken from Ref. [6].

through eq. (15). The non-linear spectra for the low-energy theory can be computed through perturbation theory, with the crucial modification that (i) all momentum integrals possess a UV cut off $k_{m}$, and (ii) the Euler equation includes pressure and viscosity contributions. The presence of the cutoff prevents the inclusion of spurious effects from the deep UV, so that fast convergence is expected. It must be emphasized that the precise value of the scale $k_{m}$ should not influence the final results for physical quantities, such as the power spectra: the explicit dependence of the effective pressure and viscosity on $k_{m}$ compensates the $k_{m}$-dependence introduced by the cutoff. In some sense, the scale $k_{m}$ may be viewed as a technical device that guarantees the good convergence of our scheme. Nevertheless, because of the perturbative truncation we expect a residual dependence that is a measure of the remaining theoretical uncertainty.

In order to test the accuracy of our results we compare them to the power spectrum extracted from $N$-body simulations [14]. In the left panel of fig. 1 we show the density power spectrum at redshift $z=0$, obtained from solving the viscous fluid equations and averaging over an initially Gaussian random distribution of the density field. The different symbols show our results obtained for the four values $k_{m}=0.4,0.6,0.8,1.0 \mathrm{~h} / \mathrm{Mpc}$. In addition, open symbols are the one-loop results, and the filled symbols show the power spectrum up to two-loops. The loop expansion is perturbative in the density contrast. However, the effect of the time-dependent pressure and viscosity on the propagation of density and velocity perturbations is taken into account non-perturbatively. We normalized all curves to the result of the $N$-body simulation. The grey-shaded area corresponds to an estimate of the error of the simulation. The agreement between $N$-body data and the two-loop results is at the percent level for $k \lesssim 0.2 \mathrm{~h} / \mathrm{Mpc}$. In addition, the variation of the results when changing the scale $k_{m}$ is mild, at the $\pm 2 \%$ level for $k \lesssim 0.2 \mathrm{~h} / \mathrm{Mpc}$. Our results can be compared to the ones obtained in standard perturbation theory (SPT) [1], depicted in the right panel of 1. For SPT we show the one- and two-loop contributions obtained when imposing a cutoff $\Lambda$ in $k$-space. The cutoff dependence in SPT is sizable at two loops, and amounts to $\pm 5 \%( \pm 10 \%)$ for $0.4 h / \mathrm{Mpc}<\Lambda<1 h / \mathrm{Mpc}(0.4 h / \mathrm{Mpc}<\Lambda \lesssim 5 h / \mathrm{Mpc})$. It is apparent that, as compared to SPT, the viscous description reduces the uncertainty generated by the treatment of UV modes significantly (left panel in fig. 1). The remaining uncertainty within 
$P_{\theta \theta}(\mathrm{k}, \mathrm{z}=0) / P_{\delta \delta}$, viscous theory

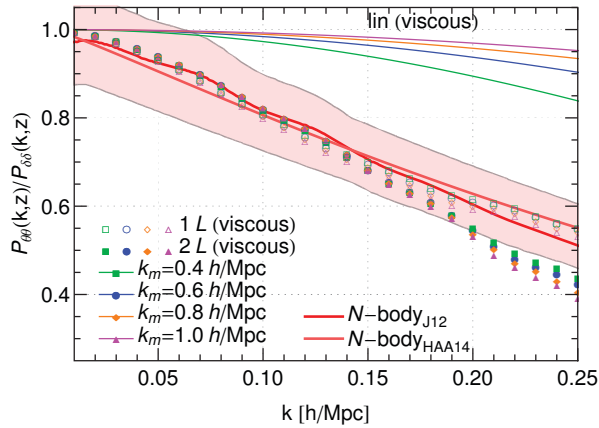

$P_{\theta \theta}(\mathrm{k}, \mathrm{z}=0) / P_{\delta \delta}, \mathrm{SPT}$ with cutoff

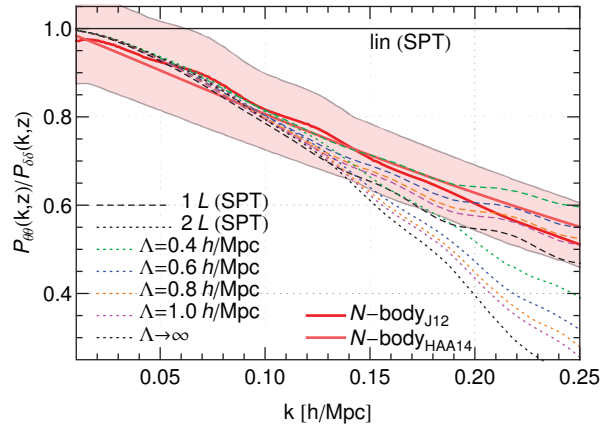

Figure 2. Power spectrum of the velocity-divergence, normalized to the density power spectrum, for the viscous theory (left) and standard perturbation theory (right). The various lines show the dependence on $k_{m}$ and on $\Lambda$, respectively, as in fig. 1. The red lines are $N$-body results ((J12) [16], (HAA14) [17]), and the shaded region is the quoted uncertainty of the velocity power spectra extracted from $N$-body data.

the viscous framework is consistent with the expected impact of effects not captured by the fluid dynamical description employed here [15].

We also consider the power spectrum for the velocity divergence and the cross power spectrum. The velocity spectrum at $z=0$ is shown in fig. 2, where we again compare the viscous description (left panel) with the result obtained in SPT (right panel) for various values of $k_{m}$ and $\Lambda$, respectively. All curves are normalized to the density power spectra corresponding to the same approximation. Fig. 3 shows a similar comparison for the cross power spectrum. The extraction of power spectra for the velocity divergence from $N$-body simulations is less robust than for the density, because of complications arising when extracting a continuous velocity field from a discrete set of particles. We show the results obtained from two different methods $[16,17]$ by the two thick red lines in figs. 2 and 3. The results are sensitive to certain parameters of the simulation, especially the resolution, and we show the uncertainty quoted in refs. $[16,17]$ as shaded regions. Within this relatively large uncertainty, the results obtained in the viscous description (left panels in figs. 2 and 3) at two loops are again in agreement up to about $k \lesssim 0.2 \mathrm{~h} / \mathrm{Mpc}$. In addition, the variation when changing $k_{m}$ is much smaller than the corresponding cutoff dependence in SPT (right panels in figs. 2 and 3). The residual $k_{m}$-dependence yields a quantitative estimate of the theoretical uncertainty. The difference of the perturbative prediction and the $N$-body results, as well as the theoretical uncertainty, increase quickly with $k$. Given that the predictions within the scheme discussed here do not require to fit any free parameters, the level of agreement between the two approaches for $k \lesssim 0.2 \mathrm{~h} / \mathrm{Mpc}$ is remarkable.

\section{Conclusions}

We conclude that the description of dark matter as a viscous fluid discussed here yields a robust framework for predicting power spectra for $k \lesssim 0.2 \mathrm{~h} / \mathrm{Mpc}$ at $z=0$ without the need to adjust any free parameters. The framework can be constructed and extended through a formal analysis based on the renormalization group. We refer the reader to ref. [8] for details. 
$P_{\delta \theta}(\mathrm{k}, \mathrm{z}=0) / P_{\delta \delta}$, viscous theory

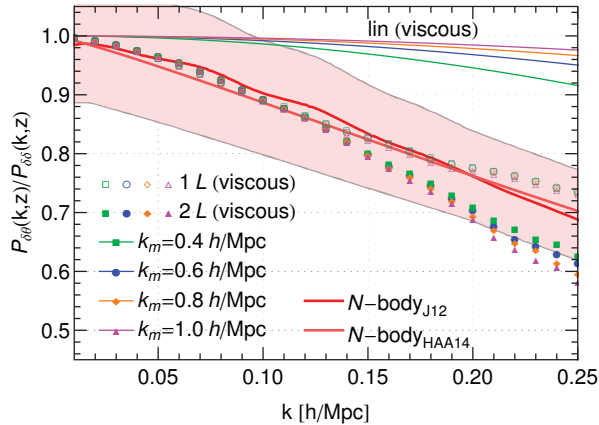

$P_{\delta \theta}(\mathrm{k}, \mathrm{z}=0) / P_{\delta \delta}, \mathrm{SPT}$ with cutoff

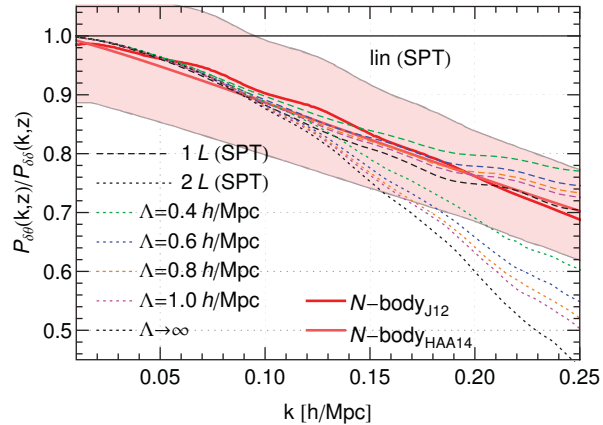

Figure 3. Cross density-velocity power spectrum, corresponding to the same approximations as in fig. 2 .

\section{References}

[1] F. Bernardeau, S. Colombi, E. Gaztanaga and R. Scoccimarro, Phys. Rept. 367 (2002) 1 [astro$\mathrm{ph} / 0112551]$.

[2] D. Baumann, A. Nicolis, L. Senatore and M. Zaldarriaga, JCAP 1207 (2012) 051 [arXiv:1004.2488 [astro-ph.CO]].

[3] J. J. M. Carrasco, M. P. Hertzberg and L. Senatore, JHEP 1209 (2012) 082 [arXiv:1206.2926 [astro-ph.CO]].

[4] M. Pietroni, G. Mangano, N. Saviano and M. Viel, JCAP 1201 (2012) 019 [arXiv:1108.5203 [astro-ph.CO]].

[5] P.J.E. Peebles, The Large-scale Structure of the Universe, Princeton University Press (1980).

[6] D. Blas, S. Floerchinger, M. Garny, N. Tetradis and U. A. Wiedemann, JCAP 1511 (2015) 049 [arXiv:1507.06665 [astro-ph.CO]].

[7] S. Matarrese and M. Pietroni, JCAP 0706 (2007) 026 [arXiv:astro-ph/0703563].

[8] S. Floerchinger, M. Garny, N. Tetradis and U. A. Wiedemann, arXiv:1607.03453 [astro-ph.CO].

[9] T. Nishimichi, F. Bernardeau and A. Taruya, arXiv:1411.2970 [astro-ph.CO].

[10] M. Garny, T. Konstandin, R. A. Porto and L. Sagunski, JCAP 1511 (2015) no.11, 032 doi:10.1088/1475-7516/2015/11/032 [arXiv:1508.06306 [astro-ph.CO]].

[11] D. B. Thomas, M. Kopp and C. Skordis, arXiv:1601.05097 [astro-ph.CO].

[12] M. Kunz, S. Nesseris and I. Sawicki, Phys. Rev. D 94 (2016) no.2, 023510 [arXiv:1604.05701 [astro-ph.CO]].

[13] M. Kopp, C. Skordis and D. B. Thomas, arXiv:1605.00649 [astro-ph.CO].

[14] J. Kim, C. Park, G. Rossi, S. M. Lee and J. R. Gott III, J. Korean Astron. Soc. 44 (2011) 217 [arXiv:1112.1754 [astro-ph.CO]].

[15] S. Pueblas and R. Scoccimarro, Phys. Rev. D 80 (2009) 043504 [arXiv:0809.4606 [astro-ph]].

[16] E. Jennings, Mon. Not. Roy. Astron. Soc. 427 (2012) L25 [arXiv:1207.1439 [astro-ph.CO]].

[17] O. Hahn, R. E. Angulo and T. Abel, Mon. Not. Roy. Astron. Soc. 454 (2015) 3920 [arXiv:1404.2280 [astro-ph.CO]]. 